\title{
Sheffield

\section{An investigation into the validation of formalised cognitive dimensions}

ROAST, C. R. <http://orcid.org/0000-0002-6931-6252> and KHAZAEI, B. Available from Sheffield Hallam University Research Archive (SHURA) at: http://shura.shu.ac.uk/18/

This document is the author deposited version. You are advised to consult the publisher's version if you wish to cite from it.

\section{Published version}

ROAST, C. R. and KHAZAEI, B. (2007). An investigation into the validation of formalised cognitive dimensions. In: Interactive systems: design, specification, and verification. Berlin, Springer, 109-122.

\section{Copyright and re-use policy}

See http://shura.shu.ac.uk/information.html 


\title{
An Investigation into the validation of formalised Cognitive Dimensions
}

\author{
Chris Roast and Babak Khazaei \\ Culture and Computing Research Centre, \\ Faculty of Arts, Computing, Engineering and Science \\ Sheffield Hallam University \\ Sheffield, S1 1WB, United Kingdom \\ c.r.roast@shu.ac.uk
}

\begin{abstract}
The cognitive dimensions framework is a conceptual framework aimed at characterising features of interactive systems that are strongly influential upon their effective use. As such the framework facilitates the critical assessment and design of a wide variety of information artifacts. Although the framework has proved to be of considerable interest to researchers and practitioners, there has been little research examining how easily the dimensions used by it can be consistently applied. The work reported in this paper addresses this problem by examining an approach to the systematic application of dimensions and assessing its success empirically. The findings demonstrate a relatively successful approach to validating the systematic application of some concepts found in the cognitive dimensions framework.
\end{abstract}

Key words: Cognitive Dimensions Framework, Theory validation, Empirical methods.

\section{Background}

The cognitive dimensions framework ([1-3]) has been developed as a simple informal, broad-brush method for evaluating information artefacts such as: notations, computer applications, and everyday devices. The essence of its approach is simple, a number of 'cognitive dimensions' are described that are properties jointly of the 'notation' and of the environment in which it is used. For instance, the dimension of 'viscosity', loosely defined as 'resistance to change', is used to characterise the ease or otherwise with which an environment and a notation allow modifications to be made. The power of the dimensions framework arises from the claim that it provides a generic basis for examining qualities that are core to the effectiveness of information artefacts. For instance, 'viscosity' as a concept can be identified in a variety of information artefact uses. Hence, the effort involved in (re-)organising ones' email folders, sub-folders and email rules can be viewed and the effort involved in, say, marking-up literary texts for automated processing both can be seen as viscous in character. Although these two examples are distinct their common characterisation enables experience within one case to be transferred to another, and so facilitating improved, and alternative, design possibilities. Inter-relations between dimensions have also been 
illustrated within the framework, for instance, in general the availability of abstraction mechanisms can relate to viscosity. An abstraction mechanism can often be used to reduce the effort that characterises high viscosity. Thus for email management the codification of relevant abstractions may be explored as a means of improving the process (as with, say, gmail). In the case of email re-organisation, the recognition of types of email, such as, request, acknowledgement, dissemination and even spam, may help reduce the effort involved in their management. In the case of literary markup, the very abstractions often embodied by logical mark-up may be explicitly supported within a tool or environment.

Unlike many frameworks associated with interface assessment and evaluation, cognitive dimensions are not intended to map out necessary improvements in a design. The framework recognises that for different problems and activities, different dimensions may be more or less relevant. The dimensions indicate the related factors that should not be ignored in design of an information artifact. Hence, whether or not a particular dimension should be minimised at the expense of another is a decision that can only be made with detailed knowledge of a particular design context. However, it is evident that for certain activities, to be successfully pursued, particular values of certain dimensions are appropriate: thus, high viscosity is not a problem for search activities, but it can be a considerable problem for modification activities.

The concepts on which the dimensions are based have received broad interest within a variety of domains where the uptake and complexity of notations and artefacts has been seen as a bottleneck in their effective exploitation. There are examples of applying the dimensions to various information artefacts, in particular: interactive systems [4], programming languages (textual and visual) [2], programming paradigms [5] and [6], design notations and specification languages [7] and [8]. In addition, various methodological techniques and tools have been developed to support their use, see [9-12].

The aim of this paper is to show that it is possible to develop formal definitions of concepts drawn from cognitive dimensions and most importantly validate the definitions with relatively a simple empirical setting.

\section{Cognitive Dimensions and their uptake}

The dimensions and the framework have on the whole been loosely defined. Green's original definitions [1] consisted of a thumbnail description followed by an example or two and this has been the style continued in later papers, such as in [2] and [3]. In particular some have argued that an informal nature means they are more readily accessible to practitioners. The ideas can be employed flexibly without demanding that procedural or semantic constraints are adhered to.

Despite this it is evident that inexperienced users of the dimensions may fail to understand what is meant by some of the concepts, and that the dimension framework can be difficult to apply in a consistent or reliable fashion. For instance, if the information artifact is complex, practitioners can be unsure as to whether dimensions have been examined to a similar level throughout. Different analysts bring a different emphasis to applying the dimensions and find it hard to systematically distinguish be- 
tween them, especially when focusing upon features and behaviours of a particular artefact. For some purposes this subjective character of the dimensions may not be a significant concern, especially if the dimensions are being employed to motivate innovative design alternatives [13]. However within the context of researching the framework and its use, ambiguities and un-certainties limit comparative assessments of artifacts and their versions, and the inter-relations between dimensions suggested by the framework.

Blandford et al [14] and Connell et al [15] provide another approach to employing cognitive dimensions in design. In this case the dimensions provide a basis for the automated analysis of a system that can be employed with only a limited account of the features and characteristics of the target system. Although the work reported below is also concerned with the automated cognitive dimensional analysis, it is motivated by the exploration of cognitive dimension definitions and their character as opposed to explicitly enabling analysis to be conducted within the context of specific design activities and case studies. Hence the definitions explored below are aimed at underpinning the type of conceptual tools that may be available for analysts and designers. The approach we take is to employ these formal definitions in the automated analysis of small scale simulated systems. With this we are able to experiment, contrasting our expectations about the cognitive dimensional character of a simulated system with those provided by following formal definitions. Experimentation with alternative definitions can also support the process of pinpointing a more objective characterisation of the cognitive dimensions and their properties.

\section{What we are doing in this paper}

With any tool there are questions that can be asked regarding whether or not it serves its intended purpose, and for cognitive dimensions this is no different. For instance, we could ask whether or not a dimension concept, such as viscosity, is valid in terms of the examples cited and the inter-relationships it has with other dimensions. Similarly as a framework we could examine its adequacy, such as whether or not design flaws that we would like it to capture can be located within the framework, or whether another dimension is required to capture important examples.

In general the validation of a tool or model involves having to bridge between its analytic domain and the empirical world in which it is intended to be valid and in which evidence is normally found. The very character of the analytic domain can complicate this process, as the analysis concepts may not have strong foundations in an empirical context. The cognitive dimensions concepts that are the focus of this work suffer from this problem. The main concepts used by the dimensions are characterised largely by means of examples and illustrations. Take for example the concept of 'viscosity': it is described in terms of the difficulty or effort when a user changes some feature or property of an interactive system, alternatively it can be viewed in terms of the systems resistance to some changes and not others. Although examples can help illustrate what 'viscosity' might be like, it is difficult to know if it is particular to types of change, objective or subjective "measures" of effort, or whether it should be viewed as absolute or relative to a specific system, activity and so forth. 
The tool that performs the automated analysis is called CiDa - given a simulated system (expressed as a state based system) and a formal definition of possible user goals and actions, CiDa performs model checking for a set of propositions about dimension properties of a system. Fig 1 outlines the relationships of interest: a simulated target system is available to both users and $\mathrm{CiDa}$ (in a form that it can analyse); the analysis of this system is provided by CiDa for users; and the value of such an analysis comes from the fact that the user is able to relate the output to their own experience with, and understanding of, the simulated system. Through experience and experimentation with the system the informal validation of the properties output by CiDa can be attempted by users.

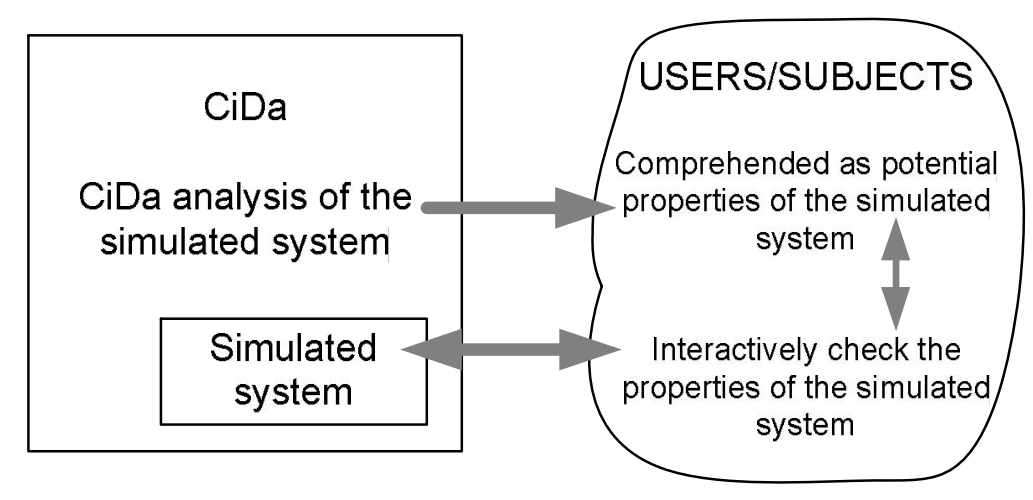

Fig. 1. The types of questions being asked of CiDa

Since CiDa provides an automated analysis it offers the advantages of being systematic and consistent in its analysis. Thus within the dimension properties defined and the potential user goals CiDa places no specific emphasis on any one dimension property or user goal, over any other.

However the effective use of CiDa for validation presupposes that the output of such an analysis can be understood and that users can satisfy themselves that it is correct. Hence in Fig 1 the block arrows should ideally represent non-problematic activities: (i) getting from $\mathrm{CiDa}$ output to an understanding of its output, (ii) users being able to relate CiDa output to how it is possible to interact and experiment with the simulated system finding the evidence for that output in system that is simulated, and (iii) engaging interactively with the system relating experience with the system back to their understanding of $\mathrm{CiDa}$ outputs. In order to assist with this process the experimental design was chosen to be one in which the simulated system was of limited scale and was represented to subjects in a "lightweight" domain setting. 


\section{An overview of CiDa and some definitions}

CiDa is a prototype model checking tool built to support theory development specifically for the cognitive dimensions framework. Although dimension formalisation have been posited [16], [5] and [6], their thorough examination and exploration is complex and can be unconvincing for practitioners. CiDa provides a model checking facility so that given proposed dimension definitions, a target system can be analysed exhaustively generating a list of dimension instances evident in the target system. The outcomes are independent of analyst expectations, bias or interests, and thus provide an objective comparable account of the dimensional features of the target system for the proposed dimension definitions. This has been of value enabling the "space" of dimensions to be explored [17] and also for proposed definitions to be enhanced. $\mathrm{CiDa}$ operates with a set of dimension definitions based mainly on temporal logic expressions, a target system definition (as a state transition system), and a bridge between the two described in terms of the possible user goals that each state of the target system may fulfill. Each definition characterises the patterns of potential goal satisfaction and actions that match the dimension of interest. Below we briefly describe the three dimensions characterisations employed in the study described.

Knock-on viscosity Although we have informally referred to 'viscosity' in the introduction, researchers in the area have found it valuable to distinguish two types of viscosity, 'knock-on' and 'repetitive' [18]. The first of these is the one examined here, it refers to the complexity, or effort, resulting from the user of a system having to make changes that have unwanted side-effects which must then be put right. This effect can be found when, say, inserting a new section in a word processed document, and having to subsequently update section number references so that the references are once again correct. Characterising this, there are two goals (a) to add a new section, and (b) to have all section references correct, there is also the action that creates the new section (act). The user's difficulty occurs when (b) has been met and now (a) has to be met as well, because adding a new section may disrupt the existing ordering. This pattern is characterised in general as follows:

In every state that can be reached:

I, goal (b) is met and goal (a) is not,

then applying act achieves goal (a) and negates goal (b)

Premature Commitment The dimension of premature commitment concerns the order in which goals can be fulfilled. Informally premature commitment occurs when a specific order of activity is demanded by a system that is in some sense inappropriate or unnecessarily constraining from the perspective of the user. More formally we employ the following concept of premature commitment based on that of [17], it is defined as a relation between two goals (a) and (b), and an action (act), such that:

1. Every behaviour leads to the goal (a) involves the action act and the other goal (b) becoming true 
2. Initially neither (a) or (b) are satisfied

3. The system behaviours ensure that (a) never necessarily entails (b) and (b) never necessarily entails (a)

Secondary Notation Besides definitions for knock-on viscosity and premature commitment $\mathrm{CiDa}$ also has a definition for the dimension of 'secondary notation'. The concept of secondary notation is that the user has a degree of freedom that enables additional alternative information to be recorded and manipulated. Within CiDa the definition of secondary notation is based upon assessing the lack of constraints that enable goals to be jointly satisfied. Fewer constraints mean that there are more potential goal combinations available to the user to employ as the basis for secondary notations.

For a primary goal (a) and a set of secondary goals (SG), the level of potential secondary notation is determined by the number of goals in $S G$ that are formally independent of satisfying (a).

\section{Experiment - assessing the CiDa analysis}

We now describe the study conducted using the prototype CiDa tool to examine whether CiDa output can be employed in empirical assessment and whether CiDa output concurs with subjects' judgments. In order to address the potential difficulties of interpretation with such a study, the following experimental guides were followed:

- CiDa output should be presented as statements that are simple and consistent in style

- The target system complexity should be low, so that subjects can in effect become experts with limited effort

- The target system domain should have familiar elements to lower the complexity of developing an understanding of it

- The target system domain should be lightweight and not strongly goal oriented so that subjects do not feel challenged by it, do not suppose that their responses are being judged, and do not presume a specific use of the system.

- Subjects are encouraged to consider the details of multiple system behaviours. This is to be encouraged by asking them to make comparative assessments of different example systems.

Five example target systems were developed and simulated for $\mathrm{CiDa}$ analysis. To allow for cross-system comparisons each provided the same functionality (i.e. the reachable state space) but differed in terms of their interactive behaviour. Hence, we were able to compare the differing systems and examine if differences were apparent in the CiDa analysis of each.

\subsection{The example systems}

The target systems used for the study were based upon the idea of simple easily ex- 
plored interactive tool for manipulating the appearance of a comic-book cartoon face. The comic-book face tools are sufficiently lightweight that novice users could easily familiarise themselves with them, and would not be unduly challenged by the domain. In addition, the graphical and direct nature of the cartoon face tools means that a realistic simulation of each design could be easily generated. All in all five alternative designs were considered, each allowed the same face configurations to be obtained, and each differed in terms of the action sequences needed to obtain face configurations

The state space consists of a range of combinations of boolean attributes: awake, unshaven, listening-to-music and talking/singing (see Fig 2). The five user actions altered the state attributes in various ways. In this guise the specific actions had no cartoon face related meaning, so arbitrary symbols with no characterisation were used (see Fig 3). It was felt the range of alternative actions and states was sufficiently limited that no authoritative explanation of the actions would be necessary for participants in the study.

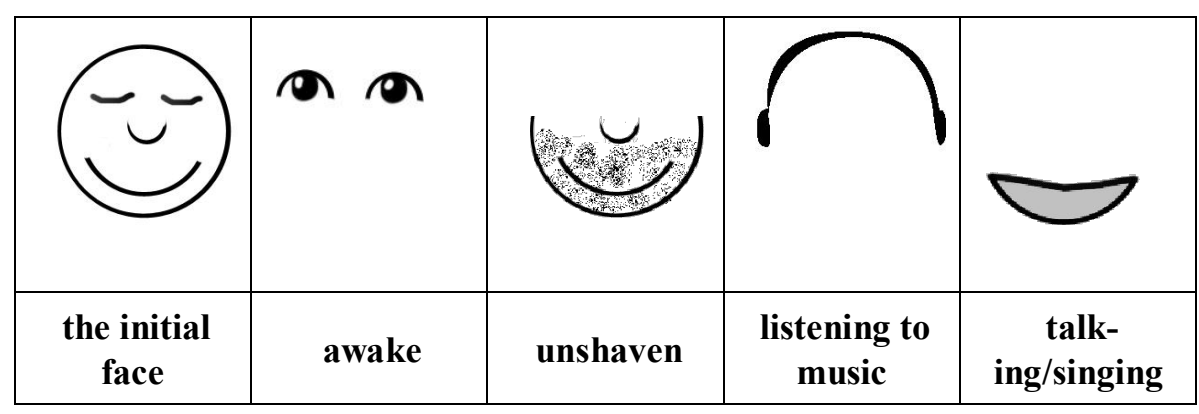

Fig. 2. The cartoon face tool with the attributes that can be present or absent.

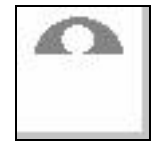

blue eye

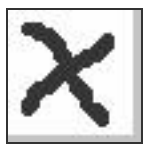

$\operatorname{red} \mathbf{X}$

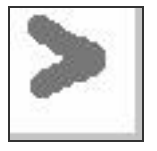

$>$

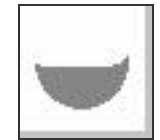

blue smile

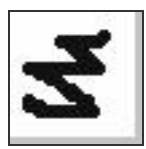

zigzag

Fig. 3. Action buttons used to control the cartoon face.

The potential user goals for this example system are listed below. The first two goals match single state attributes, the third is the explicit combination of two attributes and the last two allude to specific combinations of facial attributes.

Possible user goal - Corresponding cartoon-face attributes

Awake - Awake attribute present

Unshaven - Unshaven attribute present

Awake and clean shaven - Both awake and clean shaven attributes present

Listening late at night - headphones on present and unshaven present

Singing along - headphones on present and mouth open present 
The appendix gives details of just one of the cartoon-face tools, "Design 1", the other designs ( 2 through to 5 ) are similar but differ in their action pre-conditions. All five designs are formally defined and individually analysable by CiDa. They can also be used to simulate their corresponding version of the cartoon-face tool. It should be noted that the designs were developed to not embody a specific coherent interpretation of the actions and their effects. On the contrary, the designs were intended to limit the degree to which subjects may confidently guess at particular behaviours. Fig 4 shows an example of two of the designs in differing states, paired as used in the study.

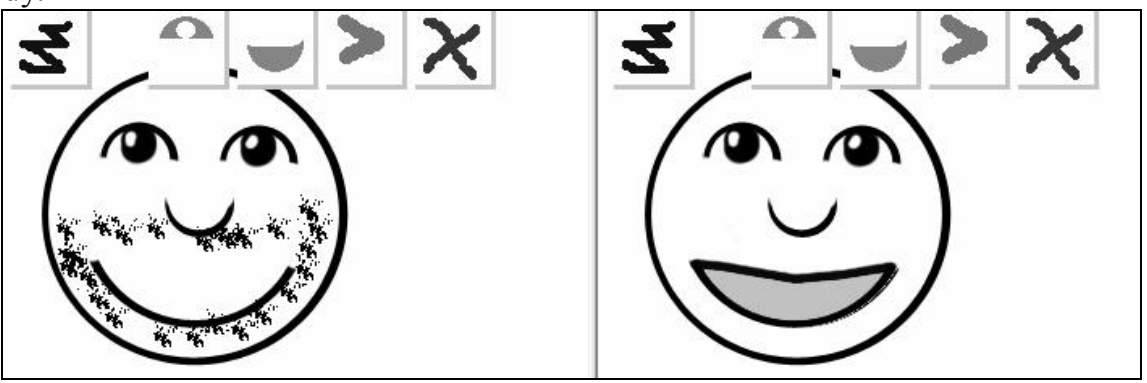

Fig. 4. A pair of the interactive cartoon face tools as used in the reported study.

\subsection{Experimental Design}

The experiment was designed to allow subjects to engage in assessing statements about the nature of the cartoon face tools. Each subject was given the task of experimenting with two of the designs and comparatively judging which design fulfilled specific dimension related statements about them. It was envisaged that providing each subject with two designs would facilitate the more accurate identification of distinctive properties and behaviours, and thus assist in their task. The statements were drawn from the cognitive dimensions instances identified in the CiDa analysis of the same set of designs. The statements were presented as written propositions largely following a template form of expression for each dimension. Below are three example statements corresponding to the three dimensions analysed:

1. If you make him so that he's not listening late (using the blue eye button), he will not be singing.

This corresponds to an instance of knock-on viscosity, making him not listen results in him not singing. Hence, if one was trying to achieve both of these, one might be frustrated by this unnecessary constraint.

2. When trying to make him chatty, you cannot avoid him being just shaved (when using the eye button).

This corresponds to an instance of premature commitment; he has to be shaved prior to chatting. Although there is nothing preventing him being unshaved and chatting.

3. You can make him unshaven and at the same time both listen to music and chat, or 
one or the other or neither.

This corresponds to an instance of secondary notation, there is sufficient flexibility in the system for the face to be unshaven while either chatting and/or listening.

To reduce the number of statements that subjects would have to assess, only a few of the possible dimension instances from CiDa's output were considered. The statements used were, where possible, ones that could be used to differentiate between the five designs used in the study. In addition, to avoid possible difficulties with comprehension, statements with relatively complex negative constructs were not used on the basis that they were likely to be more confusing and complex for subjects to assess. This resulted in a total of 12 statements for subjects to consider (see tables 1,2 and 3).

Table 1. Specific cartoon-tool statements associated with knock-on viscosity related to example designs

\begin{tabular}{|c|c|c|c|c|c|}
\hline \multirow{2}{*}{ Knock-on viscosity statements } & \multicolumn{5}{|c|}{ Design } \\
\hline & 1 & 2 & 3 & 4 & 5 \\
\hline $\begin{array}{l}\text { If you make him awake (using the blue eye button) he will be } \\
\text { shaved }\end{array}$ & Y & & & Y & \\
\hline $\begin{array}{l}\text { If you make him unshaven (using the red } \mathrm{X} \text { button) he will be lis- } \\
\text { tening late }\end{array}$ & Y & Y & & $\mathrm{Y}$ & \\
\hline $\begin{array}{l}\text { If you make him sleep (using the zigzag button) he will be clean } \\
\text { shaved }\end{array}$ & & $Y$ & $\mathrm{Y}$ & & $\mathrm{Y}$ \\
\hline $\begin{array}{l}\text { If you make him so that he's not listening late (using the blue eye } \\
\text { button), he will not be singing }\end{array}$ & $Y$ & $Y$ & $\mathrm{Y}$ & $\mathrm{Y}$ & $\mathrm{Y}$ \\
\hline $\begin{array}{l}\text { If you make him clean shaven (using the zigzag button) he will not } \\
\text { be singing }\end{array}$ & Y & Y & $\mathrm{Y}$ & Y & $\mathrm{Y}$ \\
\hline
\end{tabular}

Table 2. Specific cartoon tool statements associated with premature commitment related to example designs.

\begin{tabular}{|l|l|l|l|l|l|}
\hline \multicolumn{2}{|c|}{ Premature Commitment statements } & \multicolumn{3}{|c|}{ Design } \\
\hline $\begin{array}{l}\text { When trying to make him unshaven, you cannot avoid him being } \\
\text { shaven (when the blue eye is used) }\end{array}$ & 1 & 2 & 3 & 4 & 5 \\
\hline $\begin{array}{l}\text { When trying to make him chatty, you cannot avoid him being just } \\
\text { shaved (when using the blue eye button) }\end{array}$ & $\mathrm{Y}$ & & & $\mathrm{Y}$ & \\
\hline
\end{tabular}

Each subject was asked to experiment with randomly-allocated pairs of designs for the cartoon face tool with a view to becoming familiar with them and identifying differences between them. Fig 4 shows a pair of designs together as a subject may have seen on their screen. Following the familiarisation, the subjects were given the 12 statements on a paper questionnaire and asked to assess which was valid for which of the two designs using a five level confidence scale, ranging from definitely not valid through to definitely valid. 
A total of 25 subjects took part in the study; all had just commenced a second year undergraduate introductory human-computer interaction option, and none had received any instruction regarding the cognitive dimensions framework. Familiarisation with the example system lasted up to ten minutes. The subjects then spent around 25 minutes answering questions set. Throughout the period they were free to experiment with the two tool designs allotted to them.

Table 3. Specific cartoon tool statements associated with secondary notation potential related to example designs.

\begin{tabular}{|l|c|c|c|c|c|}
\hline \multicolumn{2}{|c|}{ Potential notation flexibility statements } & \multicolumn{3}{|c|}{ Design } \\
\hline $\begin{array}{l}\text { You can make him unshaven and at the same time both listen to } \\
\text { music and chat, or one or the other or neither }\end{array}$ & $\mathrm{Y}$ & $\mathrm{Y}$ & $\mathrm{Y}$ & $\mathrm{Y}$ & $\mathrm{Y}$ \\
\hline You can make him unshaven and the same time awake or asleep & & $\mathrm{Y}$ & $\mathrm{Y}$ & & $\mathrm{Y}$ \\
\hline $\begin{array}{l}\text { You can make him unshaven and at the same time both listen to } \\
\text { music and be awake or one or the other or neither }\end{array}$ & & $\mathrm{Y}$ & & $\mathrm{Y}$ \\
\hline $\begin{array}{l}\text { You can make him singing along and at the same time have his } \\
\text { eyes open or closed }\end{array}$ & & & $\mathrm{Y}$ & $\mathrm{Y}$ \\
\hline $\begin{array}{l}\text { You can make him not listen late and at the same time both be } \\
\text { unshaven and be awake, or either or neither }\end{array}$ & & $\mathrm{Y}$ & & $\mathrm{Y}$ \\
\hline
\end{tabular}

\subsection{Results}

Informally it was clear from the subjects' reactions to the study that not all the questions were easily answered, this was mainly evidenced from the group as a whole taking longer than planned and individuals requiring reassurance regarding details, such as whether or not their tool designs embodied different interactive behaviour. In some cases a difficulty in understanding the statements used was indicated by answers with the confidence value 3 ('Don't Know') and occasionally by their failure to answer. Missing values were assigned a rating of 3 in the analysis.

The first question in the analysis must therefore be whether some of the cognitive dimensions appeared to be harder to comprehend than others. Table 4. summarises the comparison between question types.

The frequency of answering with a 3, or leaving the question unanswered, varied little between question types, indicating that each type was equally easy (or hard) to understand. Analysis of variance showed no significant differences between question types $(\mathrm{F} 1,45=0.953)$, no interaction between question type and design $(\mathrm{F} 4,45=$ 0.337 ), and no significant difference between design types (F $4,45=0.179$ ). In general the overall low degree of indecision is a good indicator of the subjects' confidence in comprehending the questions used.

In the experiment each subject explored two designs with a view to encouraging the comparative assessment of the specific designs. A secondary element of assessing comprehension is the level of discrimination by subjects between the designs they examined. The average proportion of question responses in which subjects differenti- 
ated between designs is 0.19 and this does not differ greatly between question types: secondary notation 0.23 ; premature commitment 0.2 ; and knock-on viscosity 0.15 . This further evidences a consistent level of comprehension of the questions.

Table 4. Mean proportion of questions answered with a " 3 " or left unanswered. Figures in parentheses show standard deviations. $\mathrm{N}=50$ in each cell.

\begin{tabular}{|ll|}
\hline Question type & Mean (sd) \\
\hline secondary notation & $0.108(0.1576)$ \\
knock-on viscosity & $0.100(0.1237)$ \\
premature commitment & $0.140(0.2680)$ \\
\hline
\end{tabular}

The responses were analysed as a two-factor experiment, with repeated measures across the three question types (knock-on viscosity, premature commitment, and secondary notation) and independent groups across Designs 1 though to 5. Subjects' responses were classified as either conforming with the CiDa analysis, contrary to the $\mathrm{CiDa}$ analyis or neutral. Table 5 summarises subject agreement with respect to question type and indicates the significance of $\chi^{2}$ tests on confirming and non-confirming responses (ignoring the neutral responses).

Table 5. Classified responses for each type of question over all designs.

\begin{tabular}{|l|c|c|c|c|}
\hline Question type & Conforming & $\begin{array}{l}\text { Not } \\
\text { conforming }\end{array}$ & Significance & $\begin{array}{l}\text { Neutral } \\
\text { responses }\end{array}$ \\
\hline secondary notation & 151 & 75 & $\mathrm{p}<0.001$ & 17 \\
knock-on viscosity & 139 & 86 & $\mathrm{p}<0.001$ & 21 \\
premature commitment & 46 & 40 & $\mathrm{p}=.52$ & 7 \\
Total & 336 & 201 & $\mathrm{p}<0.001$ & 45 \\
\hline
\end{tabular}

The degree of agreement between subjects responses and $\mathrm{CiDa}$ analyses is positive and significant for two of the question types, secondary notations and knock-on viscosity. The responses to the premature commitment questions show no significant level of agreement with the $\mathrm{CiDa}$ analyses.

The differences in agreement between question types may be influenced by the differing level of experience and experimentation required by subjects to address particular questions. The statements associated with knock-on viscosity are more focused upon causal relations and, as a result, accurately answering them may require considerable investigation on the part of the subject. The statements associated with secondary notation potential are more declarative in character, and are less focused upon 
details requiring exhaustive exploration. By contrast the premature commitment type questions demand a more thorough examination of a design, implying that the subject should engage in "trying" to achieve a specific goal. This appears to be evidenced by premature commitment question type having the highest proportion of neutral responses (see table 4).

Thus although the techniques for validating CiDa output appear appropriate for secondary notation and knock-on viscosity, for premature commitment alternatives are needed, such as: offering subjects more time, re-phrasing the question templates used, etc.

\section{Conclusions}

In general, the results support the primary focus of interest, that of validating the model of the cognitive dimensions embodied in the CiDa analysis tool. The study demonstrated that employing the available domain of the graphical tools was beneficial in ensuring it was conceptually manageable for novice users, and that the method of enabling them to contrast distinct tools helped encourage their exploration. By contrast, the use of relatively dry and verbose statements relating to instances of CiDa output appears to have made the task set quite demanding. Overall, the study goes someway towards dealing with the inherent complexity of validating the effectiveness and effective use of broad-brush conceptual frameworks such as cognitive dimensions. In addition, although in some design settings formally defined properties may be viewed as unnecessarily precise, the work demonstrates that those definitions examined provide largely valid assessments.

We have shown that it is possible, important and beneficial to develop formal definitions of a usability framework based on cognitive dimensions. The potential exists to examine alternative or related formalised properties in a similar manner.

Cognitive dimensions promise great potential benefits for the effective assessment and design of complex interactive tools. There is a wide community interested in the dimensions, and the $\mathrm{CiDa}$ tool and its analysis represents one approach to strengthening the role of cognitive dimensions. The provision of a more accurate and precise framework for cognitive dimensions would benefit its employment with design and development. However, the study described here illustrates that the development and validation of a framework is not a trivial task. Despite this the results from the study are a positive step towards the validation of formalised cognitive dimensions.

Future work is to develop this approach to validation further, exploring the apparent weakness of our approach for examining premature commitment, and in general broadening the range of dimensions considered. 
Acknowledgments. The authors are indebted to Thomas Green and Simeon Yates for their comments on earlier versions of this paper and their assistance in data analysis.

\section{References}

1. Green, T. R. G.: Cognitive dimensions of notations. In A. Sutcliffe and L. Macaulay (eds.): People and Computers V. Cambridge University Press (1989)

2. Green, T. R. G., Petre, M.: Usability analysis of visual programming environments: a 'cognitive dimensions' framework. In J. Visual Languages and Computing Vol. 7(2) (1996) $131-174$

3. Green, T.R.G., Blackwell, A.F.: Design for usability using Cognitive Dimensions. Tutorial session at British Computer Society conference on Human-Computer Interaction HCI'98 (1998)

4. Roast, C.R., Dearden, A., Khazaei, B.: Enhancing contextual analysis to support the design of development tools. In Fincher, S., Markpoulos, P., Moore, D., Ruddle, R. (eds.): People and Computers XVII - Design for Life. Springer Verlag (2004)

5. Roast, C. R.: Modelling unwarranted commitment in information artefacts. In Chatty S. and Dewan P. (eds.): Engineering for Human-Computer Interaction. Kulwer Academic Press (1998) 77-90

6. Roast, C., Khazaei, B., Siddiqi, J.: Formal comparison of program modification. In Stasko J. and Pfeiffer J. J. (eds.): IEEE Symposium on Visual Languages. IEEE Computer Society (2000) 165-171

7. Britton, C., Jones, S.: The untrained eye: how languages for software specification support understanding in untrained users. In Human-Computer Interaction, Vol. 14(1) (1999) 191244

8. Khazaei, B. and Triffitt, E.: Applying Cognitive Dimensions to Evaluate and Improve the Usability of Z Formalism, Proceedings of the 14th International Conference on Software Engineering and Knowledge Engineering, ACM (2002)

9. Blackwell, A.F., Britton, C., Cox, A. Green, T.R.G., Gurr, C.A., Kadoda, G.F., Kutar, M., Loomes, M., Nehaniv, C.L., Petre, M., Roast, C., Roes, C., Wong, A., Young, R.M.: Cognitive Dimensions of Notations: design tools for cognitive technology. In M. Beynon, C.L. Nehaniv, and K. Dautenhahn (eds.): Cognitive Technology 2001 (LNAI 2117). SpringerVerlag (2001) 325-341

10. Blackwell, A.F., Green, T.R.G.: A Cognitive Dimensions questionnaire optimised for users. In Blackwell A.F. and Bilotta E. (eds.): Proceedings of the Twelfth Annual Meeting of the Psychology of Programming Interest Group. (2000) 137-152

11. Blandford, A., Green, T.R.G.: OSM an ontology-based approach to usability engineering. In Representations in Interactive Software Development. Workshop at Queen Mary and Westfield College, Department of Computer Science (1997)

12. Lavery, D., Cockton, G., Atkinson, M.: Cognitive dimensions: Usability evaluation materials. Technical report, Department of Computing Science, University of Glasgow (1996)

13. Burnett, M., Dagit, J., Lawrance, J., Beckwith, L., Kissinger, C.: Experiences with Cognitive Dimensions presented at Cognitive Dimensions of Notations 10th Anniversary Workshop (Dallas 2005)

14. Blandford, A., Connell, I., Green, T.R.G.: Concept-based Analysis of Surface and Structural Misfits (CASSM) Tutorial Notes. September 2004, available at http://www.uclic.ucl.ac.uk./annb/CASSM.html

15. Connell, I., Blandford, A., Green, T.R.G.: CASSM and cognitive walkthrough: usability issues with ticket vending machines. In Behaviour and Information Technology Vol. 23(5) (2004) $307-320$ 
16. Roast, C. R.: Formally comparing and informing design notations. In Thimbleby, H., O'Conaill, B. and Thomas, P. (eds.): People and Computers XII. Springer Verlag (1997) 315-336

17. Roast, C.R.: Dimension driven re-design - applying systematic dimensional analysis, Proceedings of the 14th Psychology of Programming Interest Group workshop (2002)

18. Green, T. R. G.: The cognitive dimension of viscosity: a sticky problem for HCI. In D. Diaper, D. Gilmore, G. Cockton and B. Shackel (eds.): Human-Computer Interaction INTERACT '90. Elsevier (1990)

\section{Appendix}

Design 1 for the cartoon face tool is represented by the state transition diagram below. The other four designs used in the study shared the same state space, but differed in terms of transitions.

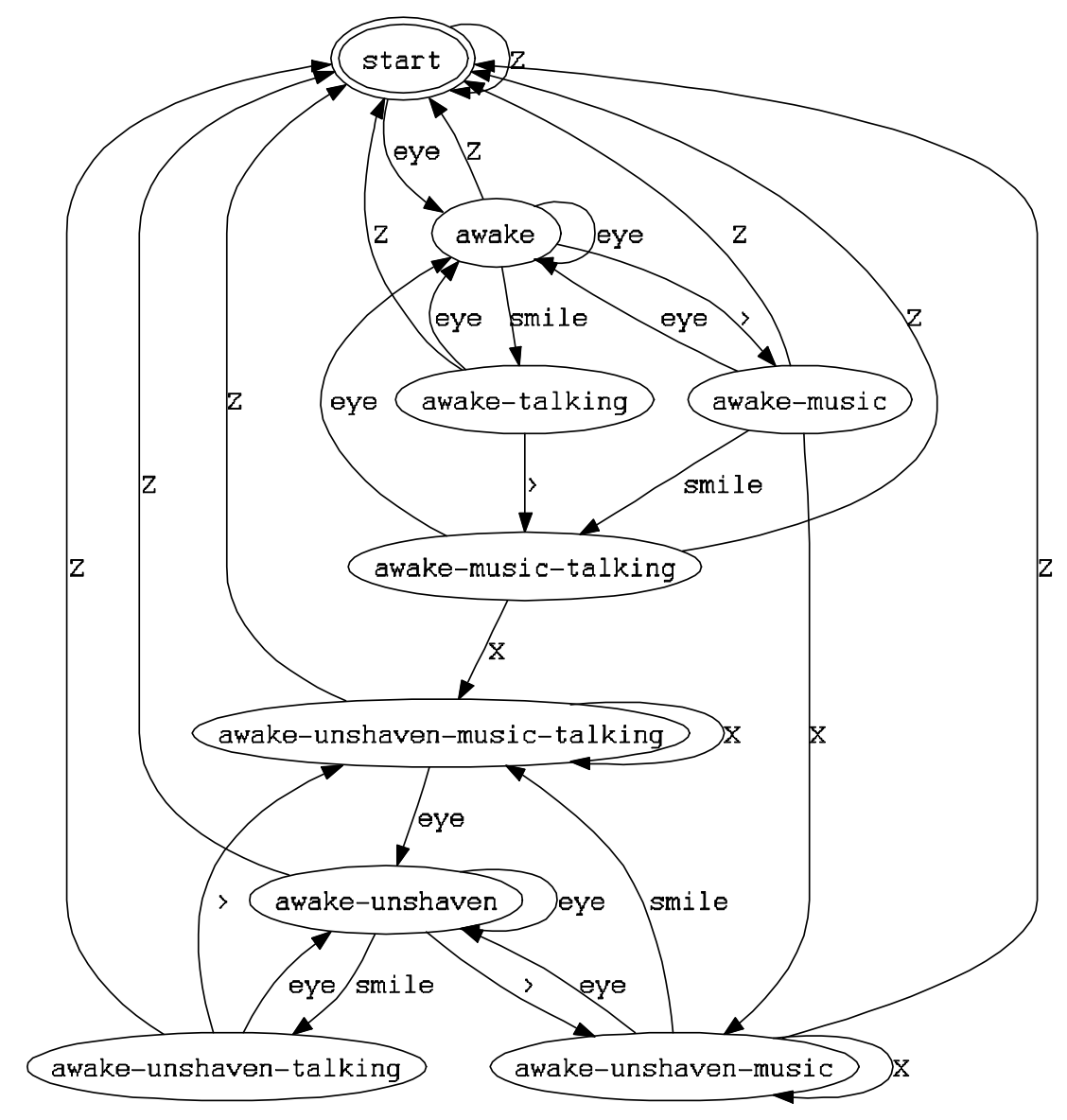

